\title{
Local lattice distortion in high-entropy alloys
}

\author{
Hongquan Song, ${ }_{1}^{1}$ Fuyang Tian, ${ }^{1, *}$ Qing-Miao Hu, ${ }^{2, \dagger}$ Levente Vitos, ${ }^{3,4,5}$ Yandong Wang, ${ }^{6}$ Jiang Shen, ${ }^{1}$ and Nanxian Chen ${ }^{1,7}$ \\ ${ }^{1}$ Institute for Applied Physics, University of Science and Technology Beijing, Beijing 100083, China \\ ${ }^{2}$ Shenyang National Laboratory for Materials Science, Institute of Metal Research, Chinese Academy of Science, Shenyang 110016, China \\ ${ }^{3}$ Applied Materials Physics, Department of Materials Science and Engineering, Royal Institute of Technology, Stockholm SE-100 44, Sweden \\ ${ }^{4}$ Department of Physics and Astronomy, Division of Materials Theory, Uppsala University, Box 516, SE-75120 Uppsala, Sweden \\ ${ }^{5}$ Institute for Solid State Physics and Optics, Wigner Research Centre for Physics, H-1525 Budapest, P.O. Box 49, Hungary \\ ${ }^{6}$ State Key Laboratory for Advanced Metals and Materials, University of Science and Technology Beijing, Beijing 100083, China \\ ${ }^{7}$ Department of Physics, Tsinghua University, Beijing 100084, China
}

(Received 15 January 2017; revised manuscript received 8 June 2017; published 19 July 2017)

\begin{abstract}
The severe local lattice distortion, induced mainly by the large atomic size mismatch of the alloy components, is one of the four core effects responsible for the unprecedented mechanical behaviors of high-entropy alloys (HEAs). In this work, we propose a supercell model, in which every lattice site has similar local atomic environment, to describe the random distributions of the atomic species in HEAs. Using these supercells in combination with abinitio calculations, we investigate the local lattice distortion of refractory HEAs with body-centered-cubic structure and $3 d$ HEAs with face-centered-cubic structure. Our results demonstrate that the local lattice distortion of the refractory HEAs is much more significant than that of the $3 d$ HEAs. We show that the atomic size mismatch evaluated with the empirical atomic radii is not accurate enough to describe the local lattice distortion. Both the lattice distortion energy and the mixing entropy contribute significantly to the thermodynamic stability of HEAs. However the local lattice distortion has negligible effect on the equilibrium lattice parameter and bulk modulus.
\end{abstract}

DOI: 10.1103/PhysRevMaterials.1.023404

\section{INTRODUCTION}

High-entropy alloys (HEAs) [1] or multicomponent alloys [2], first reported in 2004, are composed of near-equimolar metallic elements. In the last decade, many HEAs [1-4] have been discovered. The HEAs generally adopt simple single-phase crystal-lattice structures. For example, the $3 d$ HEAs [1,2] (e.g., CoFeNi, CoCrFeNi, and $\mathrm{CoCrFeMnNi}$ ) have the face-centered-cubic (fcc) structure whereas the refractory HEAs [3] (e.g., TiVNb, AlTiVNb, HfNbZr, HfNbTiZr, MoNbTaVW) have the body-centered-cubic (bcc) structure. HEAs possess interesting properties, for instance, cryogenictemperature ductility [5], superior mechanical behavior at elevated temperature [6], exceptional damage tolerance [7], superconductivity [8], as well as rich magnetic behavior [9]. The unprecedented properties are attributed to the four core effects of HEAs: high mixing entropy, severe distortion of lattice, sluggish diffusion, and cocktail effect [10-12].

The HEAs are composed of many kinds of elements with different atomic sizes. Large atoms push away their neighbors and small ones have extra space around. None of the atoms perfectly reside on the ideal lattice site. The atomic size mismatch gives rise to considerable local lattice distortions. Besides, the interatomic charge transition may also lead to local lattice distortions even though the atomic radii are close to each other.

The local lattice distortion (LLD) influences significantly the thermodynamic stability, microstructure, deformation mechanisms, and other properties of HEAs. For example, to release the lattice distortion energy induced by $\mathrm{Al}$ addition,

\footnotetext{
*fuyang@ustb.edu.cn

†qmhu@imr.ac.cn
}

the close-packed fcc structure of $\mathrm{CoCrFeNi}$ transforms to the less close-packed bcc structure, i.e., Al destabilizes the fcc structure [13]. The severe LLD reduces the growth rate of crystallites, causing the formation of nanosized or even amorphous structures. Dislocation lines in HEAs are not straight due to the local lattice distortion, which makes the deformation mechanism of HEAs different from that of the traditional alloys. The dislocation movement may be impeded by the LLD, leading to the pronounced strengthening effect. Moreover, the LLD increases the scattering of the propagating electrons and phonons, therefore, lowers the electrical and thermal conductivity of the HEAs $[14,15]$.

Evaluating quantitatively the LLD is the first step toward understanding its effect on the properties of HEAs. In literature, researchers have adopted the atomic size mismatch based on the atomic radius differences to investigate the structural features (solid solution HEAs or bulk metallic glass) [16], average residual strains [17], atomic-level stresses [18], phase stability [19], and so on. However, the definition of the atomic radius is somehow ambiguous. The same atom in different atomic environments may show different atomic radius. Therefore, an accurate and explicit description of the LLD in HEAs is desired and the atomic mismatch defined with the atomic radius needs to be carefully assessed.

First-principles methods based on density functional theory (DFT) are powerful tools to investigate the lattice distortion of alloys. By constructing a supercell model of the alloy, the equilibrium position of each atom in the supercell may be obtained through minimizing the interatomic Hellmann-Feynman forces from first-principles calculations. First-principles methods have already been used to investigate the LLD in traditional alloys (e.g., Refs. [20,21]). As for the HEAs with multiple elements, the main problem with investigating the LLD is how to construct an appropriate 
supercell in which different atoms distribute randomly. In principle, the special quasirandom structure (SQS) [22] is one of such techniques to tackle this problem. The idea of SQS is to construct a special supercell for which the atomic correlation functions mimic the physically most relevant ones of the perfectly random solid solutions. However, there are only very limited $a b$ initio studies on the local distortion based on SQS reported in literature [23,24]. Especially, to our best knowledge, no $a b$ initio investigations of the influence of local lattice distortion on the magnetism of $3 d$ HEAs have been reported so far [24].

In this work, we propose a supercell model for the random alloys. With this supercell model, we investigated the local lattice distortion and its effect on the ground-state properties (lattice parameter, bulk modulus, and magnetism) of the refractory HEAs with bcc structure and $3 d$ HEAs with fcc structure. The rest of this paper is arranged as follows: In Sec. II, we present the theoretical tool and give the most important details of the numerical calculation. The results are presented and discussed in Sec. III, and the paper ends with conclusions.

\section{SUPERCELL MODEL AND CALCULATION DETAILS}

Different from SQS, which is obtained by optimizing the correlation functions of the supercell to match those of the perfectly random alloys, the supercell model for the random alloys proposed in this work is constructed by setting the local atomic environments of all the lattice sites similar to each other. The local atomic environment is defined according to the radial distribution functions (RDF)

$$
g_{\alpha \beta}(r)=\frac{1}{N}\left\langle\sum_{i=1}^{N_{\alpha}} \sum_{j=1}^{N_{\beta}} \delta\left(r-r_{i}+r_{j}\right)\right\rangle /\left(c_{\alpha} c_{\beta} \rho\right),
$$

where $c_{\alpha}\left(c_{\beta}\right)$ is the molar fraction of alloy component $\alpha$ $(\beta) . N_{\alpha}\left(N_{\beta}\right)$ is the number of atoms of alloy component $\alpha$ $(\beta) . N$ is the total number of atoms, $\rho$ represents the overall number density, and $r$ is the interatomic distance. The supercell with similar local atomic environment (SLAE) for each lattice site may be obtained via random search. By exchanging the atomic pairs, we use the iteration scheme to approximate progressively to the SLAE condition. The periodic boundary condition satisfies the translation symmetry. The number of atomic pairs on the boundary are equally divided by the two neighboring supercells. In the SLAE supercell, the $g_{\alpha \beta}(r)$ of atomic pairs for a random alloy are proportional to the molar fraction of the corresponding alloy components.

Using the SLAE supercells, we investigate the local lattice distortion of the refractory HEAs with bcc structure and the $3 d$ HEAs with fcc structure using a first-principles planewave pseudopotential method implemented in CASTEP [25]. By computing the quantum mechanical forces and stress tensor, the supercells are fully relaxed with respect to the volume and shape of the unit cell as well as all the internal atomic positions by using Broyden-Fletcher-Goldfarb-Shanno (BFGS) optimized scheme. The convergence criteria are $10^{-5}$ $\mathrm{eV} /$ atom for the energy, $0.03 \mathrm{eV} / \AA$ for the interatomic forces, $0.05 \mathrm{GPa}$ for the stress, and $0.001 \AA$ for the displacement. The generalized gradient approximation by Perdew, Burke, and
Ernzerhof (PBE) [26] is adopted for the exchange-correlation function. We employed the on-the-fly-generation (OTFG) norm-conserving potentials. High-quality energy cutoff and dense $k$ points are chosen in order to ensure high numerical accuracy for the calculated results.

To investigate the effect of the local lattice distortion on the properties of HEAs, we calculate the bulk modulus and equilibrium volume as well as the magnetic moments and Curie temperature (for the $3 d$ HEAs) of the HEAs with and without lattice relaxation. The bulk modulus and equilibrium volume are calculated by fitting the static energy versus the volume to the fourth-order Birch-Murnaghan equation of state. The paramagnetic state (PM) above the Curie temperature is modeled by using the disordered local moment (DLM) approximation [27]. Namely, the spin-up and -down atoms with equal atomic fraction for the same elements are treated as different atomic species distributed randomly in the supercell. For example, for a $3 N$-atom ABC ternary equimolar alloy, the supercell with PM state contains six components $\left(\mathrm{A}_{0.5}^{\uparrow} \mathrm{A}_{0.5}^{\downarrow}\right)_{N}\left(\mathrm{~B}_{0.5}^{\uparrow} \mathrm{B}_{0.5}^{\downarrow}\right)_{N}\left(\mathrm{C}_{0.5}^{\uparrow} \mathrm{C}_{0.5}^{\downarrow}\right)_{N}$.

\section{RESULTS AND DISCUSSION}

\section{A. Assessing the SLAE supercells}

We construct a $3 \times 3 \times 3$ fcc or bcc SLAE supercell for the ternary equimolar HEAs, $3 \times 3 \times 4$ for the quaternary equimolar HEAs, and $3 \times 3 \times 5$ for quinary equimolar HEAs. The RDFs for pairs with up to fifth-nearest-neighboring (5NN) distance are taken into account. As two examples, in Fig. 1 we show the 36-atom hexagonal SQS structure [28] and 54-atom $3 \times 3 \times 3$ bcc SLAE supercell for the ternary HEAs.

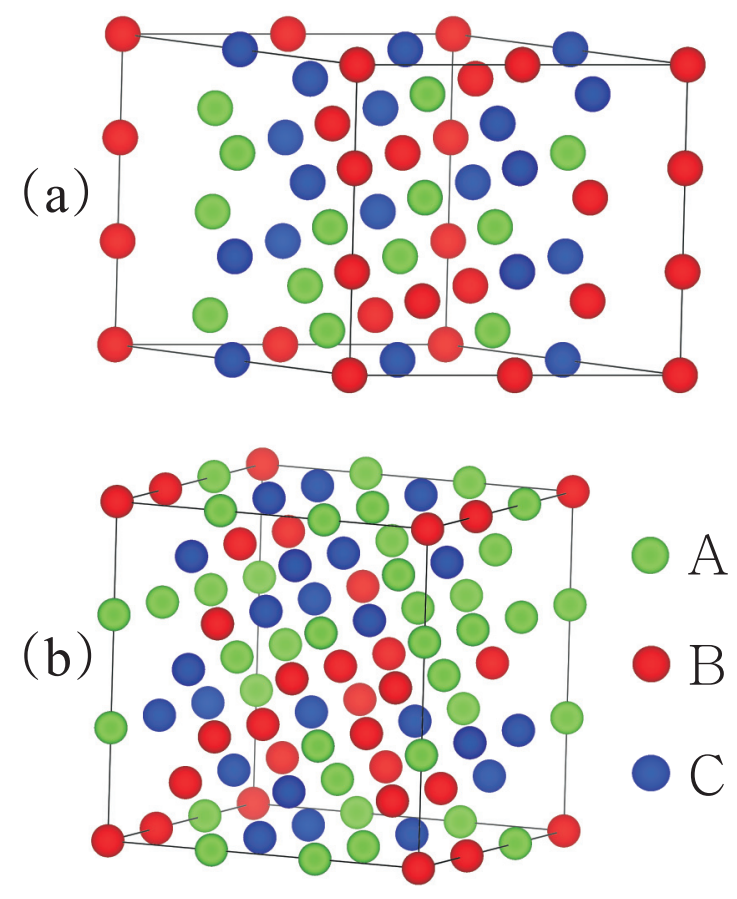

FIG. 1. The structural illustration of the 36-atom SQS (a) and the 54-atom SLAE supercell (b) for ternary bec solid solution. The SQS structure is hexagonal structure $(a=b, c / a=0.92)$. The SLAE structure is a $3 \times 3 \times 3 \mathrm{bcc}$ supercell. 


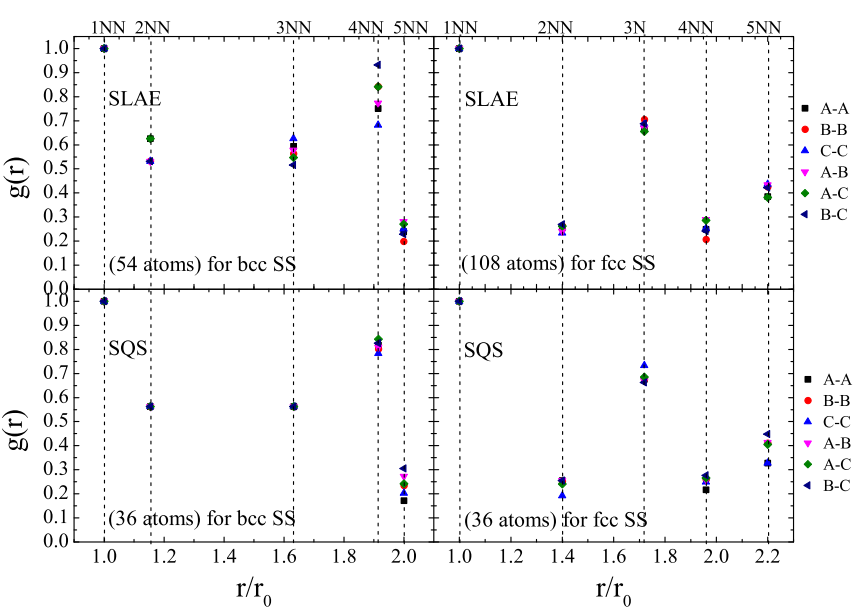

FIG. 2. The radial distribution $g(r)$ of supercells representing a ternary equimolar bcc (left panel) and fcc (right panel) solid solutions (SS). Results are shown for supercells with the similar local atomic environment (SLAE) and the special quasirandom structure (SQS) model $[28,29] . r$ is the interatomic distance and $r_{0}$ is the first-nearestneighboring $(1 \mathrm{NN})$ interatomic distance. $g(r)$ is the RDF normalized to the number of the $1 \mathrm{NN}$ interatomic pairs $N_{0}$.

For ternary equimolar HEAs, there exist six different kinds of atomic pairs, i.e., the homoatomic pairs $(A-A, B-B$, $C-C)$ and the heteroatomic pairs $(A-B, A-C$, and $B-C)$. For a perfectly random alloy, the number of different kinds of pairs [and, therefore, the RDF $g(r)$ ] with the same interatomic distance should exactly be the same. In Fig. 2 we compare the RDFs of the SLAE supercells and the 36-atom SQS supercells [28,29] for ternary equimolar HEAs. For the two supercells (SLAE and SQS), the different 1NN pairs have the same $g(r)$ values for both bcc and fcc solid solutions (SS). The $2 \mathrm{NN}$ and $3 \mathrm{NN}$ pairs in the 36 -atom SQS still have similar $g(r)$ value for bcc SS (left panel in Fig. 2), whereas in the SLAE supercell, the $g(r)$ values for different pairs are slightly different. The RDF of the 4NN pairs for the SLAE supercell are more scattered than those for the SQS, but the situation is different for the $5 \mathrm{NN}$ pairs. The discrepancy may be ascribed to the fact that the 36-atom SQS supercell was set to be noncubic in order to achieve better matching of the SQS correlation functions with those of the random alloys, whereas the SLAE supercell in this work keeps the cubic symmetry. For fcc solid solutions (right panel in Fig. 2), the $g(r)$ values for different kinds of $2 \mathrm{NN}, 3 \mathrm{NN}$, and $5 \mathrm{NN}$ pairs for the 108 -atom SLAE supercell are closer to each other than those for the 36-atom SQS. That is because that larger supercell provides more degree of freedom to fit the pair correlations between the supercell and the random alloy. For a more detailed comparison between the RDF of the SLAE and SQS supercells, one should consider several different size supercells, which is, however, beyond the scope of this work.

Figure 3 shows the RDF up to $5 \mathrm{NN}$ pairs in the SLAE supercells for quaternary and quinary equimolar HEAs with bcc and fcc structures. There exist 10 different kinds of atomic pairs for the quaternary HEAs and 15 kinds of atomic pairs for the quinary HEAs. Both quaternary bec and fcc HEAs have very close $g(r)$ values for different kinds of $1 \mathrm{NN}$ pairs. With increasing size of the supercell, the $g(r)$ values become

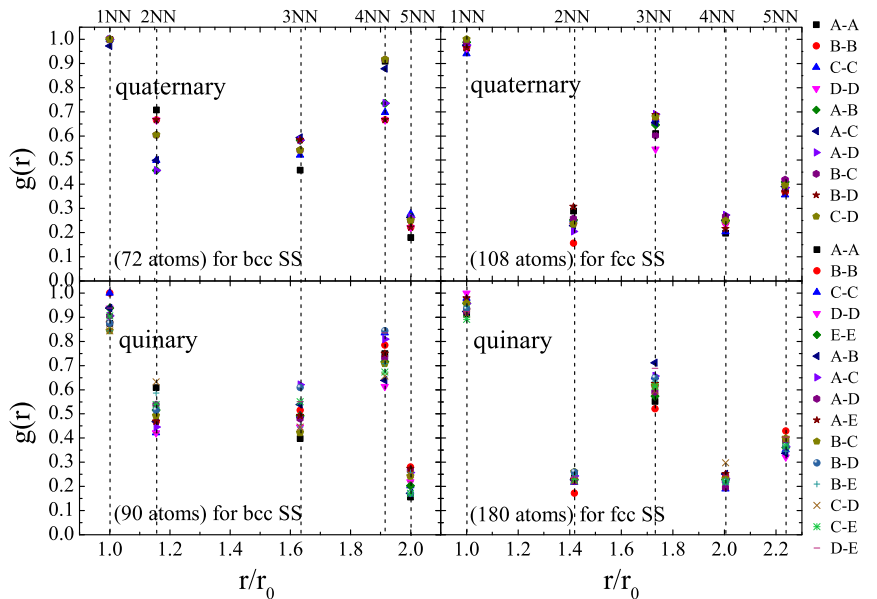

FIG. 3. The radial distribution $g(r)$ of the similar local atomic environment (SLAE) supercells representing the quaternary and quinary equimolar bcc (left panel) and fcc solid solutions (SS) (right panel). $r$ is the interatomic distance and $r_{0}$ is the first-nearestneighboring $(1 \mathrm{NN})$ interatomic distance. $g(r)$ is the RDF normalized to the number of the $1 \mathrm{NN}$ interatomic pairs $N_{0}$.

closer for the $2 \mathrm{NN}, 3 \mathrm{NN}, 4 \mathrm{NN}$, and $5 \mathrm{NN}$ pairs. Because the quaternary alloys have more kinds of pairs than the ternary alloys, the quaternary supercell show slightly more scattered $g(r)$ than the ternary alloys for the same size of the SLAE supercell (see Figs. 2 and 3).

\section{B. Degree of local lattice distortion}

The above constructed SLAE supercells are now used to investigate the local lattice distortion in HEAs. Figure 4 shows the number of atomic pairs (without distinguishing between different types of the pairs) as a function of the interatomic distance for bcc HfNbZr, HfNbTiZr, and HfNbTaTiZr. For the unrelaxed $\mathrm{HfNbZr}$, all $1 \mathrm{NN}$ pairs have the interatomic distance of $r=3.0 \AA$, and $r=3.5,4.9,5.7$, and 6.0 for the

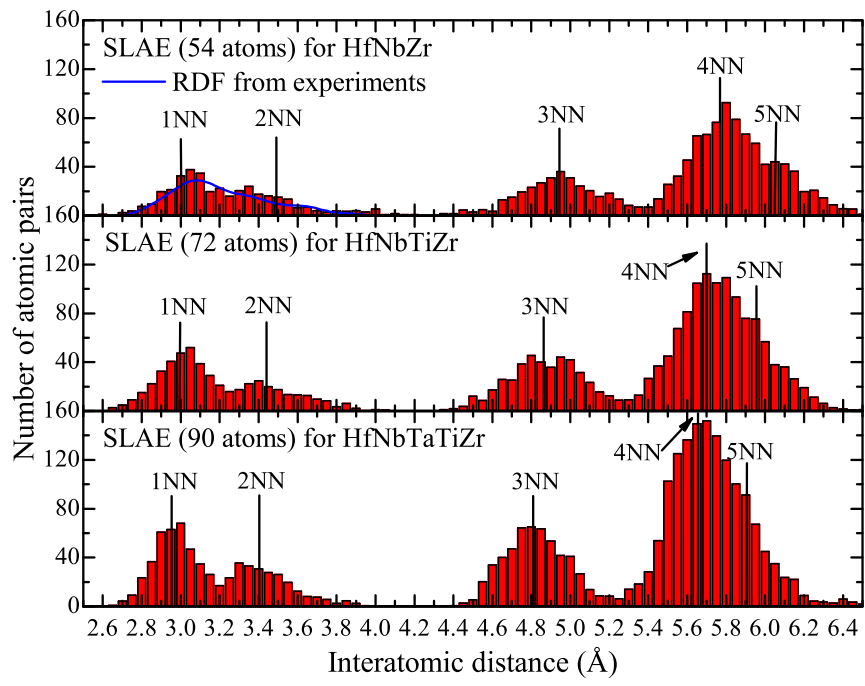

FIG. 4. Number of atomic pairs as a function of the interatomic distance in the SLAE supercells for bcc HfNbZr, HfNbTiZr, and HfNbTaTiZr. 


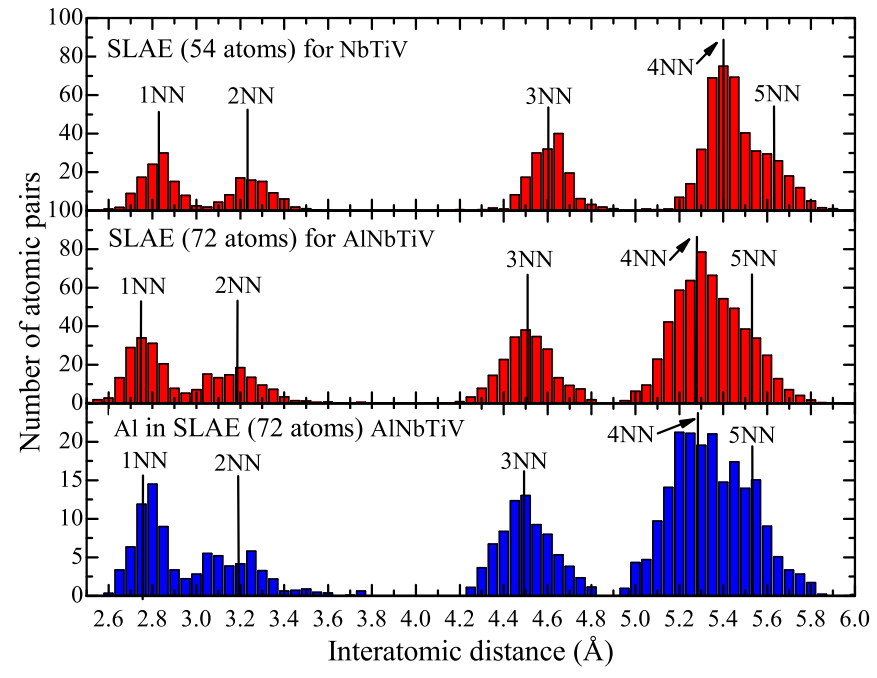

FIG. 5. Number of atomic pairs as a function of the interatomic distance in the SLAE supercells for bcc NbTiV and AlNbTiV, and degree of deviation from the ideal lattice positions near the component $\mathrm{Al}$ atoms in AlNbTiV.

$2 \mathrm{NN}, 3 \mathrm{NN}, 4 \mathrm{NN}$, and $5 \mathrm{NN}$ pairs, respectively. After atomic relaxation, the interatomic distances of these pairs spread in wide ranges. The distance gaps between the $1 \mathrm{NN}$ and $2 \mathrm{NN}$, $3 \mathrm{NN}$ and $4 \mathrm{NN}$, and $4 \mathrm{NN}$ and $5 \mathrm{NN}$ are actually filled, indicating that the atoms deviate heavily from their ideal lattice sites and severe local lattice distortion occurs. The peak positions for the maximum numbers of $1 \mathrm{NN}, 3 \mathrm{NN}$, and $4 \mathrm{NN}$ atomic pairs are still located near their ideal interatomic distances, which can be considered as the main structural feature of the HEA distinguishing from that of the amorphous phase. The predicted distribution of the atomic pairs is in good agreement with the experimental measurement [30]. With the number of atomic species increasing from $\mathrm{HfNbZr}$ to HfNbTiZr and to NfNbTaTiZr, the peaks become sharper and the interatomic distances become a little bit less spread. Namely, the local lattice distortion becomes slightly less significant with increasing number of alloy components.

Figure 5 shows the number of atomic pairs as a function of the interatomic distance for bcc NbTiV and AlNbTiV. As compared to the case of HfNbZr, HfNbTiZr, and HfNbTaTiZr, the LLDs of the NbTiV and AlNbTiV are less significant. The separation between $3 \mathrm{NN}$ and $4 \mathrm{NN}$ pairs for $\mathrm{NbTiV}$ and AlNbTiV are much wider than those for HfNbZr, HfNbTiZr, and HfNbTaTiZr. Unlike HfNbZr, HfNbTiZr, and HfNbTaTiZr, in NbTiV the LLD increases upon adding another alloying element (Al). Considering the strong bonding between $\mathrm{Al}$ and the refractory metals, we show the radial distribution near $\mathrm{Al}$ atoms in Fig. 5. The results suggest that Al has large magnitude of deviation from ideal pairs, which suggests that $\mathrm{Al}$ induces large local lattice distortion in the refractory alloys.

Figure 6 shows the number of pairs as a function of interatomic distance for the ternary $\mathrm{CoFeNi}$, quaternary $\mathrm{CoCrFeNi}$, and quinary CoCrFeMnNi $3 d$ fcc HEAs. The peaks for the maximum number of $1 \mathrm{NN}, 2 \mathrm{NN}, 3 \mathrm{NN}, 4 \mathrm{NN}$, and $5 \mathrm{NN}$ atomic pairs are all located quite sharply near their ideal interatomic

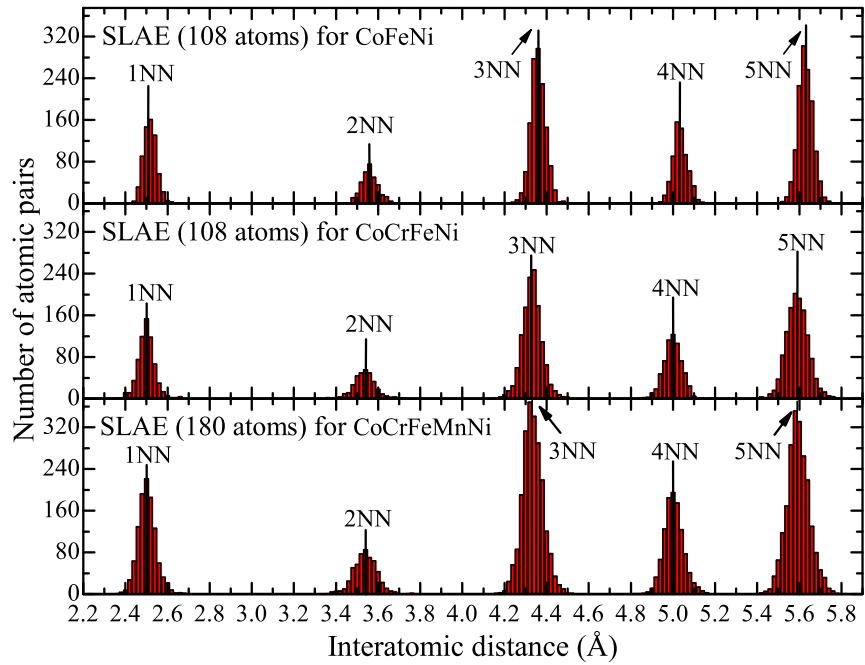

FIG. 6. Number of atomic pairs as a function of the interatomic distance in the SLAE supercells for fcc CoFeNi, CoCrFeNi, and $\mathrm{CoCrFeMnNi}$.

distances, indicating that the local lattice distortion is much weaker in fcc HEAs than that in the refractory bcc HEAs.

As we introduced in Sec. I, the atomic size mismatch was frequently adopted to measure the LLD effects in HEAs. The atomic size mismatch [31] is generally estimated as

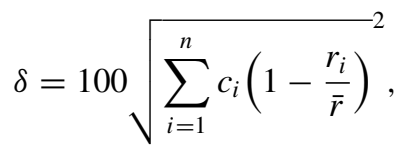

where $\bar{r}=\sum_{i=1}^{n} c_{i} r_{i}$, with $c_{i}$ and $r_{i}$ being the atomic percentage and atomic radius of the individual alloy component, respectively. Such an estimation is somehow ambiguous because the same atomic species in different systems may show different atomic radii and there are serval different definitions of the atomic radii. The atomic radii of the atoms may be measured by using x-ray diffraction in their elemental states, which have been used to investigate the glass-forming ability of amorphous metallic alloys by Senkov et al. [32]. On the other hand, the atomic radii may be derived from the 12-coordinated system [33]. Therefore, it is interesting to evaluate the accuracy of the atomic size mismatch with different definitions of the atomic radius for the description of the local lattice distortion in HEAs. We adopt the quantity

$$
\Delta d=\frac{1}{N} \sum_{i} \sqrt{\left(x_{i}-x_{i}^{\prime}\right)^{2}+\left(y_{i}-y_{i}^{\prime}\right)^{2}+\left(z_{i}-z_{i}^{\prime}\right)^{2}}
$$

to describe the local lattice distortion in HEAs. Here $\left(x_{i}, y_{i}\right.$, $\left.z_{i}\right)$ and $\left(x_{i}^{\prime}, y_{i}^{\prime}, z_{i}^{\prime}\right)$ are the reduced coordinates of the unrelaxed and relaxed positions of atom $i$, respectively. The $\delta-\Delta d$ data points for different HEAs are plotted in Fig. 7.

From Fig. 7, we see that the atomic size mismatch $\delta$ and the calculated local lattice distortion $\Delta d$ are in reasonable agreement with each other: larger $\delta$ usually corresponds to larger $\Delta d$. However, for some alloys $\delta$ calculated with the elemental or the 12-coordinated atomic radii cannot predict correctly the local lattice distortions. In particular, NbTiV has a quite large $\delta$ but a relatively small $\Delta d$ and, as a result, NbTiV 


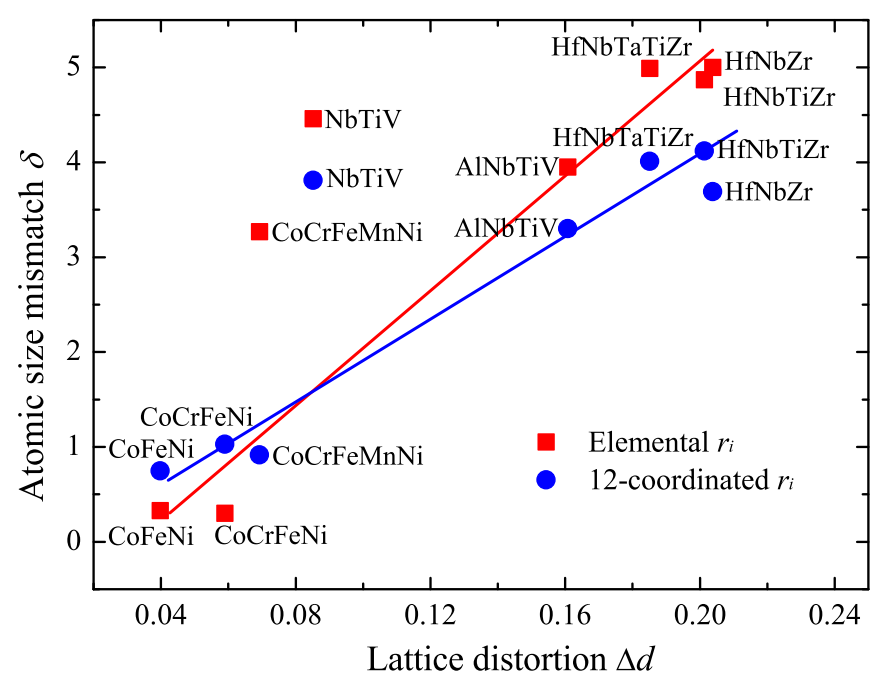

FIG. 7. Atomic size mismatch $\delta$ evaluated with the elemental [32] and 12-coordinated [33] atomic radius differences versus the $a b$ initio predicted local lattice distortion $\Delta d$ [see Eq. (3)] for bcc HfNbZr, HfNbTiZr, HfNbTaTiZr, NbTiV, and AlNbTiV and for fcc $\mathrm{CoFeNi}, \mathrm{CoCrFeNi}$, and $\mathrm{CoCrFeMnNi}$.

deviates significantly from the near linear $\delta-\Delta d$ relationship for the other HEAs. The same is true for $\mathrm{CoCrFeMnNi}$ with $\delta$ calculated with the elemental $r_{i}$. The atomic size mismatch $\delta$ calculated with elemental $r_{i}$ slightly overestimates the local lattice distortions of the refractory HEAs but slightly underestimated those of the $3 d$ HEAs as compared to $\delta$ calculated with the 12-coordinated $r_{i}$. Notably, the difference of the atomic size mismatches of $\mathrm{CoCrFeMnNi}$ calculated with the elemental and 12-coordinated $r_{i}$ 's is much larger than those of the other HEAs.

\section{Lattice distortion energy and phase stability}

For the refractory HEAs, the lattice distortion energy $\Delta E_{\text {dis }}$, defined as the difference between the energies calculated without and with atomic relaxation, decreases from $7.776 \mathrm{~kJ} / \mathrm{mol}$ for $\mathrm{HfNbZr}$ to $6.216 \mathrm{~kJ} / \mathrm{mol}$ for HfNbTiZr to $4.232 \mathrm{~kJ} / \mathrm{mol}$ for HfNbTaTiZr (see Table I). This trend is in agreement with the decreasing local lattice distortion in the same order as discussed in Sec. III B. The lattice distortion energies of the refractory HEAs are in general substantially higher than those for the $3 d$ HEAs ranging from $0.392 \mathrm{~kJ} / \mathrm{mol}$ for $\mathrm{CoCrFeMnNi}$ to $1.858 \mathrm{~kJ} / \mathrm{mol}$ for CoCrFeNi. In Table I we also report a few $\mathrm{SQS}$ results for comparison. It can be seen that the distorted energy from SLAE is consistent with that from SQS for the ternary HfNbZr and NbTiV HEAs.

The mixing enthalpy $\Delta H_{\mathrm{mix}}$ is defined as the difference between the energy of HEAs, $E_{\mathrm{HEA}}$, and the ground-state energies of the alloy components $E_{i}$ :

$$
\Delta H_{\mathrm{mix}}=E_{\mathrm{HEA}}-\sum_{i} c_{i} E_{i}
$$

where $c_{i}$ the atomic fraction of the alloy component $i$. Table I lists the mixing enthalpy with and without LLD. The mixing enthalpy calculated without LLD is positive for most HEAs expected for MoNbTaVW and AlNbTiV. Upon taking into account the local lattice distortion, the mixing enthalpy decreases for all HEAs by $\Delta E_{\text {dis }}$, i.e., the local lattice distortion stabilizes the solid solutions of HEAs. However, the mixing enthalpy for most of the HEAs is still positive, indicating these HEAs could actually be stabilized by entropy effects.

The mixing entropy is another important contribution to the stability of solid solutions. For a totally random alloy, the mixing entropy is calculated as

$$
\Delta S_{\text {mix }}=k_{B} \sum_{i} c_{i} \ln c_{i},
$$

where $k_{B}$ is the Boltzmann constant. For equimolar HEAs, the above equation reduces to $\Delta S=k_{B} \ln N$, where $N$ is the number of alloy components. At room temperature $(T=$ $300 \mathrm{~K}$ ), the contribution of the mixing entropy to the free energy, $-T \Delta S_{\text {mix }}$, is $-2.739 \mathrm{~kJ} / \mathrm{mol}$ for the ternary equimolar alloy, $-3.459 \mathrm{~kJ} / \mathrm{mol}$ for the quaternary equimolar alloy, and $-4.014 \mathrm{~kJ} / \mathrm{mol}$ for the quinary equimolar alloy. The Gibbs free energies $G=\Delta H_{\mathrm{mix}}-T \Delta S_{\mathrm{mix}}$ are also listed in Table I. It turns out that all present HEAs have negative Gibbs free energy, except NbTiV. When compared to the relaxation energy, we find that the mixing entropy has similar magnitude as $\Delta E_{\text {dis }}$ and becomes dominant only in quinary bec and all fcc HEAs. It is clear that without a proper account for the local lattice relaxation, no meaningful Gibbs energy of formation can be predicted.

\section{Effect of LLD on lattice parameters and bulk modulus}

To investigate the effect of the local lattice distortion on the properties of HEAs, we calculate the Wigner-Seitz radius, the entire set of lattice parameters (allowing for lattice distortion upon relaxation), and the bulk modulus of the HEAs with and without atomic relaxations. The results are listed in Table II.

TABLE I. Lattice distortion energy $\Delta E_{\text {dis }}(\mathrm{kJ} / \mathrm{mol})$, mixing enthalpy $\Delta H_{\text {mix }}(\mathrm{kJ} / \mathrm{mol})$ with and without local lattice distortions, contribution of the mixing entropy to the free energy $-T \Delta S_{\text {mix }}(\mathrm{kJ} / \mathrm{mol})$ at $T=300 \mathrm{~K}$, and the Gibbs free energy $G(\mathrm{~kJ} / \mathrm{mol})$ of the present ternary, quaternary, and quinary HEAs.

\begin{tabular}{lrcccrrrrr}
\hline \hline HEA & HfNbZr & HfNbTiZr & HfNbTaTiZr & MoNbTaVW & NbTiV & AlNbTiV & CoFeNi & CoCrFeNi & CoCrFeMnNi \\
\hline$\Delta E_{\text {dis }}$ (SLAE) & 7.776 & 6.216 & 4.232 & 2.736 & 2.166 & 4.090 & 1.267 & 1.858 & 0.392 \\
$\Delta E_{\text {dis }}$ (SQS) & 6.988 & & & & 2.880 & & & \\
$\Delta H_{\text {mix }}$ (no LLD) & 10.156 & 9.426 & 6.895 & -10.628 & 7.178 & -4.964 & 1.632 & 0.525 \\
$\Delta H_{\text {mix }}$ (LLD) & 2.380 & 3.210 & 2.663 & -13.364 & 5.012 & -9.053 & 0.375 & -1.333 & 0.674 \\
$-T \Delta S_{\text {mix }}$ & -2.739 & -3.459 & -4.014 & -4.014 & -2.739 & -3.459 & -2.739 & -3.459 & -4.014 \\
$G$ & -0.341 & -0.249 & -1.351 & -17.378 & 2.273 & -12.512 & -2.364 & -4.792 & -3.340 \\
\hline \hline
\end{tabular}


TABLE II. Wigner-Seitz (WS) radius $r_{0}(\AA)$, bulk modulus $B(\mathrm{GPa})$ and its pressure derivative $B^{\prime}$, and lattice parameters $(a, b, c \AA, \alpha, \beta$, and $\gamma$ in degree) of the present HEAs without and with local lattice relaxation. The available experimental Wigner-Seitz radii are listed for comparison. $\delta r$ stands for the relative error of WS radius for the present EHAs without and with local lattice relaxation.

\begin{tabular}{|c|c|c|c|c|c|c|c|c|c|c|c|}
\hline HEA & $r_{0}$ & $\delta r$ & $r_{\text {expt }}$ & $B$ & $B^{\prime}$ & $a$ & $b$ & $c$ & $\alpha$ & $\beta$ & $\gamma$ \\
\hline $\mathrm{HfNbZr}$ & 1.717 & & & 111.6 & 3.725 & 10.400 & 10.400 & 10.400 & 90.0 & 90.0 & 90.0 \\
\hline distorted & 1.707 & $-0.6 \%$ & $1.717[30]$ & 116.2 & 3.738 & 10.420 & 10.560 & 10.430 & 90.1 & 90.2 & 89.6 \\
\hline HfNbTiZr & 1.685 & & & 110.5 & 3.295 & 10.268 & 10.268 & 13.691 & 90.0 & 90.0 & 90.0 \\
\hline distorted & 1.693 & $0.5 \%$ & $1.733[34]$ & 112.9 & 3.412 & 10.304 & 10.382 & 13.684 & 89.9 & 90.0 & 89.7 \\
\hline HfNbTaTiZr & 1.673 & & & 124.1 & 3.390 & 10.193 & 10.193 & 16.989 & 90.0 & 90.0 & 90.0 \\
\hline distorted & 1.677 & $0.2 \%$ & $1.676[35]$ & 125.4 & 3.663 & 10.316 & 10.166 & 16.995 & 90.3 & 90.1 & 90.0 \\
\hline MoNbTaVW & 1.573 & & & 212.7 & 4.000 & 9.585 & 9.585 & 15.975 & 90.0 & 90.0 & 90.0 \\
\hline distorted & 1.572 & $-0.1 \%$ & $1.567[3]$ & 217.4 & 3.973 & 5.590 & 5.594 & 15.944 & 90.1 & 90.0 & 89.8 \\
\hline NbTiV & 1.572 & & & 151.8 & 3.596 & 9.584 & 9.584 & 9.584 & 90.0 & 90.0 & 90.0 \\
\hline distorted & 1.573 & $0.1 \%$ & & 152.0 & 3.717 & 9.606 & 9.569 & 9.556 & 90.2 & 89.9 & 90.1 \\
\hline AlNbTiV & 1.568 & & & 134.5 & 3.542 & 9.558 & 9.558 & 12.744 & 90.0 & 90.0 & 90.0 \\
\hline distorted & 1.570 & $0.1 \%$ & $1.566[19]$ & 134.7 & 3.012 & 9.511 & 9.710 & 12.600 & 90.0 & 89.9 & 90.3 \\
\hline $\mathrm{CoFeNi}$ & 1.391 & & & 179.5 & 3.644 & 10.678 & 10.678 & 10.678 & 90.0 & 90.0 & 90.0 \\
\hline distorted & 1.392 & $0.1 \%$ & 1.406 [9] & 182.1 & 3.671 & 10.646 & 10.699 & 10.693 & 90.1 & 90.1 & 90.0 \\
\hline $\mathrm{CoCrFeNi}$ & 1.379 & & & 172.7 & 6.681 & 10.584 & 10.584 & 10.584 & 90.0 & 90.0 & 90.0 \\
\hline distorted & 1.381 & $0.2 \%$ & $1.393[36]$ & 181.8 & 5.494 & 10.566 & 10.666 & 10.583 & 90.2 & 90.0 & 89.9 \\
\hline $\mathrm{CoCrFeMnNi}$ & 1.382 & & & 178.3 & 5.143 & 10.594 & 10.594 & 10.726 & 90.0 & 90.0 & 90.0 \\
\hline distorted & 1.383 & $0.1 \%$ & 1.403 [2] & 180.1 & 5.321 & 10.602 & 10.665 & 17.609 & 90.0 & 90.1 & 90.0 \\
\hline
\end{tabular}

For all HEAs considered here, the local lattice distortion has minor effect on the lattice parameters. The largest error between the lattice parameters of the refractory HEAs calculated with and without local lattice distortion is about $-0.6 \%$ and it is about $0.1 \%$ for the $3 d$ HEAs. The lattice angles of the supercells of the HEAs are close to $90^{\circ}$ after fully relaxation of the supercells, i.e., the supercell deviate only slightly from cubic. The calculated Wigner-Seitz radii are in good agreement with experimental measurements.

The bulk moduli of all the HEAs calculated with atomic relaxation are close to those calculated without atomic relaxation. The largest difference is only about $5 \%$ for $\mathrm{HfNbZr}$, MoNbTaVW, and CoCrFeNi. Hence, the bulk modulus of the present HEAs is not sensitive to the local lattice distortion either.

The insensitivity of the equilibrium volume $\left(V_{0}=\frac{4}{3} \pi r_{0}^{3}\right)$ and bulk modulus to the local lattice distortion was also found for the simple solid solutions such as fcc- $\mathrm{Ti}_{1-x} \mathrm{Al}_{x}$ [20] and fcc- $\mathrm{Cu}_{1-x} \mathrm{Au}_{x}$ [21]. This indicates that the equilibrium volume and bulk modulus are mainly determined by the compositions of the alloys and the first-principles methods in combination with the mean-field approximation such as coherent potential approximation to describe the randomness of the alloy can be safely used to predict these properties $[37,38]$.

\section{E. Effect of LLD on magnetic properties}

The alloy components of $3 d$ HEAs are magnetic in their ground state: $\mathrm{Fe}, \mathrm{Co}$, and $\mathrm{Ni}$ are ferromagnetic (FM), i.e., the directions of the magnetic moments on all lattice sites in the ground state are the same, Mn has multimagnetic state, and $\mathrm{Cr}$ is antiferromagnetic (AM), i.e., magnetic moments of the two sublattices are opposite to each other. For the present $3 d$ HEAs, the FM state is more stable than the PM state at $0 \mathrm{~K}$. We should notice that the FM state of the present quaternary and quinary are actually ferrimagnetic since $\mathrm{Cr}$ and $\mathrm{Mn}$ have opposite moments compared to the rest of the alloy components.

With increasing number of alloy elements, the average magnetic moment per atom decreases from $1.697 \mu_{B}$ for $\mathrm{CoFeNi}$ to $0.641 \mu_{B}$ for $\mathrm{CoCrFeNi}$, and to $0.334 \mu_{B}$ for $\mathrm{CoCrFeMnNi}$ (see Table II). This trend is in good agreement with those calculated by using first-principles exact muffintin orbital (EMTO) method in combination with coherent potential approximation $\left(1.67 \mu_{B}, 0.66 \mu_{B}\right.$, and $0.23 \mu_{B}$ for the present HEAs), respectively [40]. There are at least two reasons for the decrease in average magnetic moment. First, $\mathrm{Cr}$ and $\mathrm{Mn}$ favor the antiferromagnetic alignment and thus decrease the total magnetic moment. The average local magnetic moments of $\mathrm{Cr}$ and $\mathrm{Mn}$ are about $\bar{\mu}_{\mathrm{Cr}}=-0.74 \mu_{B}$ in $\mathrm{CoCrFeNi}, \bar{\mu}_{\mathrm{Cr}}=$ $-0.29 \mu_{B}$, and $\mu_{\mathrm{Mn}}=-0.57 \mu_{B}$ in CoCrFeMnNi. Second, the local magnetic moments of $\mathrm{Fe}, \mathrm{Co}, \mathrm{Ni}$ decrease with the addition of $\mathrm{Cr}$ and $\mathrm{Mn}$. The local magnetic moments of $\mathrm{Fe}, \mathrm{Co}, \mathrm{Ni}$ decrease from $\bar{\mu}_{\mathrm{Fe}}=2.68, \bar{\mu}_{\mathrm{Co}}=1.72, \bar{\mu}_{\mathrm{Ni}}=$ $0.70 \mu_{B}$ in CoFeNi to $\bar{\mu}_{\mathrm{Fe}}=2.07, \bar{\mu}_{\mathrm{Co}}=0.97, \bar{\mu}_{\mathrm{Ni}}=0.28 \mu_{B}$ in $\mathrm{CoCrFeNi}$, to $\bar{\mu}_{\mathrm{Fe}}=1.74, \bar{\mu}_{\mathrm{Co}}=0.66, \bar{\mu}_{\mathrm{Ni}}=0.14$ in $\mathrm{CoCrFeMnNi}$.

Listed in Table III is also the Curie temperature $T_{C}$ estimated from the energy difference between the FM and PM states calculated with the local lattice distortion. In line with the decreased magnetic moments, the Curie temperature $T_{C}$ also decreases from $\mathrm{CoFeNi}$ to $\mathrm{CoCrFeNi}$ and to $\mathrm{CoCrFeMnNi}$. We notice that unfortunately the Curie temperatures for the undistorted HEAs are not presented since we failed to have the PM calculations converging to the same accuracy. On the other hand, the Curie temperature of the undistorted HEAs has been calculated by using the first-principles EMTO in combination with the coherent potential approximation [39], These former results are listed in Table III for reference. It is seen that the CPA- $T_{C}$ 's of $\mathrm{CoFeNi}$ and $\mathrm{CoCrFeNi}$ 
TABLE III. Listed are the average magnetic moment per atom $\left(\mu_{B}\right)$ for ferromagnetic $\mathrm{CoFeNi}, \mathrm{CoCrFeNi}$, and $\mathrm{CoCrFeMnNi}$ HEAs, and Curie temperature $T_{C}(\mathrm{~K})$ of $\mathrm{CoFeNi}, \mathrm{CoCrFeNi}$, and $\mathrm{CoCrFeMnNi.}$

\begin{tabular}{lccc}
\hline \hline HEA & $\mu_{B}$ & $T_{C}$ & $T_{C}(\mathrm{CPA})$ \\
\hline CoFeNi & 1.685 & & 868 [39] \\
distorted & 1.697 & 717.8 & \\
CoCrFeNi & 0.659 & & 156 [39] \\
distorted & 0.641 & 81.3 & 23 [39] \\
CoCrFeMnNi & 0.324 & & \\
distorted & 0.334 & 31.4 & \\
\hline \hline
\end{tabular}

are higher than the corresponding values calculated in this work with local lattice distortion, whereas it is opposite for $\mathrm{CoCrFeMnNi}$. The discrepancy of $T_{C}$ might be partly ascribed to the local lattice distortion that was omitted in the CPA calculations. However, for a robust verdict, one should perform unrelaxed calculations using the same formalism. The experimental Curie temperature is $119 \mathrm{~K}$ for $\mathrm{CoCrFeNi}$, in general agreement with $81.3 \mathrm{~K}$ from our calculations and also with $156 \mathrm{~K}$ reported previously in EMTO calculations.

\section{CONCLUSION}

We have proposed a supercell model with similar local atomic environment (SLAE) to simulate a random solid solution with multiple elements. The supercells were used to study the local lattice distortion in refractory and $3 d$ HEAs.
In the SLAE supercell every lattice site possesses similar radial distribution functions ensuring the similar neighboring environment. Our results demonstrate that the local lattice distortion of the refractory HEAs is much more significant than that of the $3 d$ HEAs. The atomic size mismatch evaluated with the atomic radius is not accurate enough to predict the local lattice distortion effects. Both the lattice distortion energy and the mixing entropy contribute substantially to the stability of the HEAs and only in quaternary and quinary bcc alloys and in all fcc alloys becomes the mixing entropy dominant. The local lattice distortion does not affect significantly the lattice parameter and bulk modulus. The present developments offer an alternative opportunity to study multicomponents HEAs using $a b$ initio methods and confirm the importance of local lattice distortion effects especially in less close-packed alloys.

\section{ACKNOWLEDGMENTS}

Work was supported by the Science Challenge Project (Grant No. JCKY2016212A502), the National Natural Science Foundation of China (Grant No. 51401014), the Foundation of Key Laboratory of Neutron Physics, CAEP (Grant No. 2015BB04), the National Key Basic Research Program (973) (Grants No. 2011CB606401 and No. 2014CB644001), and the National Key Research and Development Project of China (Grant No. 2016YFB0701301). L.V. acknowledges financial support by the Swedish Research Council, the Swedish Foundation for Strategic Research, the Swedish Foundation for International Cooperation in Research and Higher Education, the Carl Tryggers Foundation, and the Hungarian Scientific Research Fund (OTKA 109570).
[1] J. W. Yeh, S. K. Chen, S. J. Lin, J. Y. Gan, T. S. Chin, T. T. Shun, C. H. Tsau, and S. Y. Chang, Adv. Eng. Mater. 6, 299 (2004).

[2] B. Cantor, I. T. H. Chang, P. Knight, and A. J. B. Vincent, Mater. Sci. Eng. A 375-377, 213 (2004).

[3] O. N. Senkov, G. B. Wilks, D. B. Miracle, C. P. Chuang, and P. K. Liaw, Intermetallics 18, 1758 (2010).

[4] A. Takeuchi, K. Amiya, T. Wada, K. Yubuta, and W. Zhang, JOM 66, 1984 (2014).

[5] B. Gludovatz, A. Hohenwarter, D. Catoor, E. H. Chang, E. P. George, and R. O. Ritchie, Science 345, 1153 (2014).

[6] Y. Zou, H. Ma, and R. Spolenak, Nat. Commun. 6, 7748 (2015).

[7] B. Gludovatz, A. Hohenwarter, K. V. S. Thurston, H. Bei, Z. Wu, E. P. George, and R. O. Ritchie, Nat. Commun. 7, 10602 (2016).

[8] P. Koželj, S. Vrtnik, A. Jelen, S. Jazbec, Z. Jagličić, S. Maiti, M. Feuerbacher, W. Steurer, and J. Dolinšek, Phys. Rev. Lett. 113, 107001 (2014).

[9] Y. Zhang, T. Zuo, Y. Cheng, and P. K. Liaw, Sci. Rep. 3, 1455 (2013).

[10] Y. Zhang, T. Zuo, Z. Tang, M. Gao, K. Dahmen, P. Liaw, and Z. Lu, Prog. Mater. Sci. 61, 1 (2014).

[11] R. Kozak, A. Sologubenko, and W. Steurer, Z. Kristallogr. 230, 55 (2015).

[12] E. J. Pickering and N. G. Jones, Int. Mater. Rev. 61, 183 (2016).
[13] Y.-F. Kao, T.-J. Chen, S.-K. Chen, and J.-W. Yeh, J. Alloys Compd. 488, 57 (2009).

[14] D. B. Miracle and O. N. Senkov, Acta Mater. 122, 448 (2017).

[15] Z. Fan, H. Wang, Y. Wu, X. Liu, and Z. Lu, Mater. Res. Lett. 5 , 187 (2017).

[16] S. Guo and C. Liu, Prog. Nat. Sci.: Mater. Int. 21, 433 (2011).

[17] Y. Ye, C. Liu, and Y. Yang, Acta Mater. 94, 152 (2015).

[18] T. Egami, W. Guo, P. D. Rack, and T. Nagase, Metall. Mater. Trans. A 45, 180 (2014).

[19] N. D. Stepanov, D. G. Shaysultanov, G. A. Salishchev, M. A. Tikhonovsky, E. E. Oleynik, A. S. Tortika, and O. N. Senkov, J. Alloys Compd. 628, 170 (2015).

[20] L.-Y. Tian, Q.-M. Hu, R. Yang, J. Zhao, B. Johansson, and L. Vitos, J. Phys.: Condens. Matter 27, 315702 (2015).

[21] L.-Y. Tian, L.-H. Ye, Q.-M. Hu, S. Lu, J. Zhao, and L. Vitos, Comput. Mater. Sci. 128, 302 (2017).

[22] A. Zunger, S.-H. Wei, L. G. Ferreira, and J. E. Bernard, Phys. Rev. Lett. 65, 353 (1990).

[23] I. Toda-Caraballo, J. S. Wróbel, S. L. Dudarev, D. NguyenManh, and P. E. J. Rivera-Díaz-Del-Castillo, Acta Mater. 97, 156 (2015).

[24] H. Oh, D. Ma, G. Leyson, B. Grabowski, E. Park, F. Körmann, and D. Raabe, Entropy 18, 321 (2016).

[25] S. J. Clark, M. D. Segall, C. J. Pickard, P. J. Hasnip, M. I. J. Probert, K. Refson, and M. C. Payne, Z. Kristallogr. 220, 567 (2005). 
[26] J. P. Perdew, K. Burke, and M. Ernzerhof, Phys. Rev. Lett. 77, 3865 (1996).

[27] B. L. Gyorffy, A. J. Pindor, J. Staunton, G. M. Stocks, and H. Winter, J. Phys. F: Met. Phys. 15, 1337 (1985).

[28] C. Jiang, Acta Mater. 57, 4716 (2009).

[29] L. Vinet and A. Zhedanov, J. Phys. A: Math. Theor. 44, 085201 (2011).

[30] W. Guo, W. Dmowski, J.-Y. Noh, P. Rack, P. K. Liaw, and T. Egami, Metall. Mater. Trans. A 44, 1994 (2013).

[31] Y. Zhang, Y. J. Zhou, J. P. Lin, G. L. Chen, and P. K. Liaw, Adv. Eng. Mater. 10, 534 (2008).

[32] O. N. Senkov and D. B. Miracle, Pergamon Mater. Res. Bull. 36, 2183 (2001).

[33] W. Pearson, Crystal Chemistry and Physcis of Metals and Alloys (Wiley, New York, 1972).
[34] Y. Wu, Y. Cai, T. Wang, J. Si, J. Zhu, Y. Wang, and X. Hui, Mater. Lett. 130, 277 (2014).

[35] O. Senkov, J. Scott, S. Senkova, D. Miracle, and C. Woodward, J. Alloys Compd. 509, 6043 (2011).

[36] M. S. Lucas, L. Mauger, J. A. Muñoz, Y. Xiao, A. O. Sheets, S. L. Semiatin, J. Horwath, and Z. Turgut, J. Appl. Phys. 109, 07E307 (2011).

[37] F. Tian, L. K. Varga, N. Chen, L. Delczeg, and L. Vitos, Phys. Rev. B 87, 075144 (2013).

[38] F. Tian, L. K. Varga, N. Chen, J. Shen, and L. Vitos, J. Alloys Compd. 599, 19 (2014).

[39] F. Körmann, D. Ma, D. D. Belyea, M. S. Lucas, C. W. Miller, B. Grabowski, and M. H. F. Sluiter, Appl. Phys. Lett. 107, 142404 (2015).

[40] F. Tian, L. K. Varga, J. Shen, and L. Vitos, Comput. Mater. Sci. 111, 350 (2016). 